\title{
AUTORITARISMO E BENEVOLENCIA FRENTE A DOENÇA MENTAL: ESTUDO COM ALUNOS INGRESSANTES NO CURSO DE ENFERMAGEM
}

Rita de Cássia Avanci ${ }^{1}$

Silmara Elaine Malaguti ${ }^{2}$

Luiz Jorge Pedrão ${ }^{3}$

Avanci RC, Malaguti SE, Pedrão LJ. Autoritarismo e benevolência frente à doença mental: estudo com alunos ingressantes no curso de enfermagem. Rev. Latino-am Enfermagem 2002 julho-agosto; 10(4):509-15.

Alunos iniciantes no curso de Enfermagem tendem a exibir atitudes negativas frente ao portador de doença mental assim como se manifesta, de forma geral, a população. Este estudo objetivou estudar o autoritarismo e a benevolência desses alunos frente a esse paciente e, para isso, foi utilizada a escala de opiniões sobre a doença mental. Os resultados apontaram que os alunos em questão mostraram-se autoritários frente ao referido paciente, com tendência a um protecionismo bondoso, permitindo concluir que esses aspectos devem ser considerados na elaboração dos programas das disciplinas dessa área, para levá-los a condutas mais terapêuticas no futuro.

DESCRITORES: enfermagem psiquiátrica, educação em enfermagem, atitude

\section{AUTHORITARIANISM AND BENEVOLENGE TOWARDS MENTAL ILLNESS: STUDY WITH STUDENTS BEGINNING A NURSING PROGRAM}

Students beginning a nursing program tend to show negative attitudes towards mental health patientsm as does the general population. This study aimed at studying these students' authoritarianism and benevolence towards mental patients utilizing the opinion scale about the mental illness. The results indicated that the students showed authoritarianism towards mental patients with a tendency to protective behavior, which enabled the conclusion that these aspects must be considered in the elaboration of the course programs in this area in order to lead them to more therapeutic conducts in the future.

DESCRIPTORS: psychiatric nursing, nursing education, attitude

\section{AUTORITARISMO Y BENEVOLENCIA FRENTE A LA ENFERMEDAD MENTAL: ESTUdIO CON ALUMNOS QUE INGRESAN AL CURSO DE ENFERMERÍA}

Los alumnos principiantes del curso de enfermería tienden a exhibir actitudes negativas frente al Portador de enfermedad mental como la población en general. Este estudio tiene como objetivo el autoritarismo y la benevolencia de estas alumnos frente a estos pacientes, y por este motivo fue utilizada una escala de opiniones sobre la enfermedad mental. Los resultados demostraron que los alumnos en cuestión se mostraban autoritarios frente al paciente con enfermedad mental con tendencia a un proteccionismo bondadoso, permitiendo concluir que estos aspectos deben ser considerados en la elaboración de los programas de las disciplinas de esta área, para llevarlas a conductas más terapéuticas en un futuro.

DESCRIPTORES: enfermería psiquiátrica, educación en enfermería, actitud

\footnotetext{
${ }^{1}$ Aluna de Graduação em Enfermagem, Bolsista PIBIC/USP/CNPq 1998 e 1999; ${ }^{2}$ Aluna de Graduação em Enfermagem; ${ }^{3}$ Professor Doutor, email: lujope@eerp.usp.br. Escola de Enfermagem de Ribeirão Preto, da Universidade de São Paulo, Centro Colaborador da OMS para o desenvolvimento da pesquisa em enfermagem
} 


\section{INTRODUÇÃO}

A psiquiatria, desde que surgiu, no final do século XVIII, como um novo campo do saber, vem se transformando e, acompanhando todo seu movimento, a enfermagem psiquiátrica também se transforma. No Brasil, essa área específica da enfermagem surgiu no hospício, com o objetivo de vigiar, controlar e reprimir, perfil que se manteve durante muito tempo, pois os seus profissionais não conseguiam mudar o modo de entender e assistir o doente mental, o qual continuava sendo visto como louco e perigoso. Mesmo o surgimento de novos hospitais psiquiátricos, tanto públicos quanto privados, e ambulatórios, não conseguiu mudar a assistência psiquiátrica $^{(1)}$, levando a um entendimento de que a mudança no modo de compreender, aceitar e assistir, de forma adequada, o referido doente, antecede à criação ou ampliação de serviços assistenciais.

Pode-se dizer que as mudanças na enfermagem psiquiátrica brasileira começaram a partir da Lei 775/49 sobre o ensino de enfermagem, que determinava a obrigatoriedade da disciplina "Enfermagem Psiquiátrica" nos cursos de graduação em enfermagem. No entanto, as ações de enfermagem psiquiátrica eram muito tímidas, aparecendo nos programas apenas algumas aulas sobre a assistência de enfermagem voltada a diversos transtornos psiquiátricos ou sobre os psicofármacos, cuja indústria começava a se expandir ${ }^{(2)}$.

As reais mudanças na assistência de enfermagem psiquiátrica começaram a partir do final da década de 60 , quando suas ações começaram a ter base no relacionamento terapêutico influenciado tanto pela Psiquiatria Social, que fez críticas ao modelo asilar e propôs a sua transformação no sentido de tornar-se um ambiente onde a saúde mental pudesse emergir, quanto pela Comunidade Terapêutica, que considerou fundamental o treinamento da equipe, o tipo de relacionamento que se estabelece na comunidade e o papel ativo e responsável que o paciente é estimulado a assumir ${ }^{(1-2)}$.

Dessa forma, no que diz respeito à transformação da enfermagem psiquiátrica, vários autores concordam com que o papel do enfermeiro psiquiátrico é de agente terapêutico, e suas ações têm base no relacionamento estabelecido com o paciente psiquiátrico, a partir da compreensão do significado de seu comportamento e da sua aceitação como ser humano. É claro que isso implica, o enfermeiro psiquiátrico dispor de conhecimento científico e habilidade profissional e pessoal, para desempenhar esse papel ${ }^{(3-8)}$.

Assim sendo, as disciplinas de enfermagem psiquiátrica e de enfermagem em saúde mental, como as demais disciplinas da área de ciências humanas do curso de graduação em enfermagem, devem ter os seus programas voltados a esses aspectos. Devem, inclusive, estar atentas para as várias mudanças que continuam ocorrendo no modo de assistir a pessoa portadora de doença mental, pois entende-se que a prática de enfermagem custodial começa a ser parte de um passado e que o aluno pretendente ao exercício específico da enfermagem psiquiátrica passa a necessitar de embasamentos que permitam que ele atue de maneira ativa e eficaz em um ambiente terapêutico e seja um agente terapêutico num contexto de trabalho multidisciplinar.

Portanto, o enfermeiro que deseja inserir-se no mercado de trabalho, na área específica de enfermagem psiquiátrica, necessita de conhecimentos que o levem a condutas terapêuticas, frente ao paciente com o qual trabalha, e que possam conferir-lhe uma posição de destaque na equipe multidisciplinar por ser um observador privilegiado (presente 24 horas/dia) e deter o maior número de informação sobre esse paciente, contribuindo, assim, não só para o planejamento de cuidados, mas também na organização da parte estrutural de uma unidade para assistência psiquiátrica.

Então, aquele papel autoritário de vigiar, controlar e reprimir, característico do início da enfermagem psiquiátrica brasileira e desenvolvido no hospício ${ }^{(1)}$, é substituído por um outro papel, que é o terapêutico, por meio do relacionamento terapêutico interpessoal, e, para isso, o autoritarismo tem a necessidade de ser trabalhado, pois uma atitude terapêutica firme, objetiva e com apoio no saber, é muito diferente de uma atitude autoritaria no sentido restritivo e discriminador, assim como atitudes benevolentes no sentido paternalista. Esse papel do enfermeiro psiquiátrico, em busca ainda de uma definição mais adequada, exige desse profissional um melhor conhecimento de si, pois conhecendo e controlando melhor as suas emoções, mais chances terá de entender e trabalhar as emoções de outro, sendo, então, ele próprio a ferramenta de trabalho no contexto interpessoal ${ }^{(8-10)}$.

Observamos, assim, que atitudes autoritárias e benevolentes podem interferir negativamente nas 
condutas que o enfermeiro psiquiátrico tem com seu paciente portador de doença mental. Estudar como esses dois aspectos ocorrem em alunos ingressantes no curso de graduação em enfermagem é particularmente importante, tendo em vista que podem ser trabalhados de maneira mais objetiva durante todo o curso, preparando-o melhor para oferecer não só uma assistência em enfermagem psiquiátrica como em saúde mental mais terapêutica e eficiente, não somente dentro de hospitais ou unidades psiquiátricas fechadas, mas também em modalidades de assistência aberta, buscando o cuidado integral do portador de doença mental citado, desestimulando, assim, o cuidado dividido em tarefas ${ }^{(11)}$.

Em se tratando de atitude, cabe lembrar que, para sua investigação sobre um determinado objeto, doença ou situação, é necessário, a princípio, conceituar este termo, tendo em vista a não coincidência absoluta sobre a sua definição por diferentes autores ${ }^{(12)}$.

Com relação à enfermagem, a atitude do enfermeiro pode ser definida como procedimentos realizados com o propósito de preparar o cliente para os trabalhos clínicos e orientar o seu acompanhante, para que possa colaborar na sua readaptação ao seu meio familiar e social. A atitude terapêutica não é produto de mero instinto, mas sim reações comportamentais que uma vez formadas por um profissional, deveriam ser incorporadas ao seu modo de ação ${ }^{(13-14)}$.

São inúmeras as definições propostas para atitude, mas, mesmo com as mais variadas interpretações, existem áreas que exibem uma concordância essencial, e grande parte dos autores concordariam que uma atitude pode ser descrita como "predisposição" para responder, de maneira consistente favorável ou desfavorável, em relação a um dado "objeto". Entretanto, existem dúvidas quanto à consistência das respostas, e, devido a isso, a maioria dos instrumentos, para sua medida, tem base na consistência avaliativa ou afetiva, sendo que uma pessoa, em diferentes situações, pode exibir diferentes comportamentos em relação a um dado objeto, mas a favorabilidade ou a desfavorabilidade expressa por esses comportamentos, mantém-se constante. A consistência afetiva permite diferenciar uma atitude de outros conceitos como hábito, traço, impulso, etc $^{(12,15)}$.

Da mesma forma que ocorre com "atitude", é necessário definir "doença mental", tendo em vista ser ela um fenômeno cuja maneira de entendimento varia amplamente, em diferentes culturas e comunidades, apresentando, ainda, variações com o passar do tempo ${ }^{(16)}$.

Considerando os questionamentos que surgem quando há a necessidade de se definir "dificuldade emocional", "doença mental" ou "transtorno psiquiátrico" e se diferentes populações compartilham, similarmente, tais definições, o estudo realizado pela World Federation of Mental Health ${ }^{(17)}$, parece ser um dos mais amplos por se tratar de uma investigação transcultural. Esse estudo mostra que a população, em geral, não distingue os vários tipos de distúrbios mentais. Difere, apenas, o esgotamento nervoso da doença mental, classificando, na primeira categoria, os distúrbios originados do meio ambiente e que, submetidos a um tratamento de curta duração, teriam "cura". A segunda categoria, mais complexa, afetaria o indivíduo em todos os níveis de desempenho, necessitaria de internações de longa duração, não proporcionando uma recuperação completa do paciente, pelo fato de sua personalidade já ter um comprometimento permanente.

Podem-se completar essas considerações com as de vários autores, que referem ser o grau de desvio das normas sociais aceitas, prescritas pela sociedade, maior do que a patologia profissionalmente diagnosticada $^{(18)}$. Em outras palavras, vale dizer que a tolerância da sociedade para com a pessoa portadora de doença mental é desproporcional em sentido desfavorável, à real ameaça que essa pessoa é para esta sociedade.

A população, de forma geral, exibe noções sobre a doença e o doente mental do tipo racionalista (fatores psíquicos ou orgânicos) ou tradicionalista (cérebro fraco), sendo a primeira apresentada por indivíduos que desempenham atividades ocupacionais não manuais, e a última, apresentada por indivíduos que desenvolvem atividades ocupacionais manuais. Estereótipos e preconceitos, como pessoa boba que não raciocina; agressiva, estranha; perigosa e que nunca mais sara; que traz problemas para a família e que tem que ficar no hospício, são exibidos também pela população, sem uma correspondência real com os comportamentos que esses doentes emitem no meio social ${ }^{(19)}$.

Alunos iniciantes no Curso de Graduação em Enfermagem trazem consigo estereótipos e preconceitos semelhantes aos descritos anteriormente, e chegam a 
definir o doente mental como "velho e burro", demonstrando total desconhecimento, tanto com relação à incidência da doença mental nas várias faixas etárias, quanto com relação à recuperação desse paciente e ainda das possibilidades de sua convivência no seu meio social e familiar.

Esses estereótipos e preconceitos podem influenciar, de forma negativa, as condutas que esses alunos terão com o paciente em questão e, no futuro, enquanto profissionais, se esses aspectos não foram trabalhados adequadamente durante sua formação, têm grandes chances de traduzirem-se em atitudes autoritárias e benevolentes (paternalistas) e ainda outras, implicando no agir de modo não terapêutico com o paciente referido.

Parte-se do princípio, também, de que atitudes frente à doença mental e ao seu portador, de forma geral, são passíveis de ser modificadas, principalmente em estudantes da área da saúde, quando submetidos à instrução acadêmica ${ }^{(12)} \mathrm{e}$, em particular, no estudante de enfermagem, durante o seu aprendizado teórico-prático nas disciplinas de enfermagem psiquiátrica e de enfermagem em saúde mental, devido ao intenso contato com o paciente citado e, ainda, na observação dos professores durante suas abordagens e interações demonstrativas com esses pacientes, momento em que ele se coloca, também, como um modelo de referência a ser seguido pelo aluno.

Assim, oferecer subsídios para o planejamento e o desenvolvimento das disciplinas citadas anteriormente, constitui-se no principal propósito do presente estudo, verificando-se a presença de autoritarismo e benevolência (paternalismo) de alunos ingressantes no curso de enfermagem, frente à doença mental e à pessoa em sofrimento psíquico, antes deles terem contato com as disciplinas referidas.

\section{METODOLOGIA}

Sujeitos e local

Participaram deste estudo 68 alunos matriculados no primeiro semestre do Curso de Graduação em Enfermagem e Obstetrícia da Escola de Enfermagem de Ribeirão Preto, da Universidade de São Paulo (EERP-USP), no ano de 1998.
Escala de avaliação utilizada

Foi utilizada a escala de atitudes - Opiniões Sobre a Doença Mental - ODM ${ }^{(20)}$, traduzida e validada para o nosso meio ${ }^{(17)}$. Essa escala é composta de 51 afirmações do tipo LIKERT, e suas opções de respostas variam segundo uma seqüência progressiva de seis pontos de concordância, desde "concordo totalmente" até "discordo totalmente". Essas afirmações foram agrupadas em sete fatores ${ }^{(16)}$ dos quais foram extraídos os relativos ao autoritarismo e a benevolência, para a finalidade deste estudo, que foram definidos ${ }^{(17)}$ da seguinte forma:

- Autoritarismo - reflete a perspectiva de que o doente mental necessita ser isolado de outros pacientes, permanecendo sob portas trancadas e vigilância. Contém tanto o conceito da irrecuperabilidade pessoal e social do doente quanto a idéia de sua periculosidade.

- Benevolência - traduz a visão de que o doente mental, devido a sua infelicidade, deve ser amparado por meio de um protecionismo bondoso e paternalista, com base em cuidados, atenção pessoal e conforto material.

Procedimento

Aplicação da ODM

Inicialmente, em sala de aula, os oitenta alunos matriculados no primeiro semestre do Curso de Graduação em Enfermagem e Obstetrícia, da EERP/USP, foram convidados, de forma verbal, a responderem à escala ODM. Marcamos uma data para a aplicação desta escala, em um dia da semana, 40 minutos antes do início da aula de uma disciplina, quando todos os alunos estavam reunidos, uma vez que 68 aceitaram o convite para participar.

Foi feito contato com o docente que ministrava a referida disciplina no dia escolhido, no sentido de comunicar-lhe a realização da atividade e também pedir sua colaboração, caso houvesse necessidade de tolerância no horário para início de sua aula.

Após terem respondido, a escala foi recolhida e arquivada para futura análise.

A aplicação da escala aos alunos foi realizada pelos autores do presente estudo, que puderam também, no momento, esclarecer possíveis dúvidas.

Análise dos resultados

Para contagem dos pontos obtidos com a 
aplicação da ODM, foram utilizadas fórmulas ${ }^{(21)}$, originando, assim, os escores fatoriais brutos para cada aluno, nos fatores referentes ao autoritarismo e à benevolência dessa escala. Posteriormente, esses escores foram transformados para o Sistema Sten, que se mostra adequado, pois proporciona uma comparação padrão entre os fatores da escala referida, com variação entre 1 e 10, média de 5,5 e um desvio padrão igual a $0,5^{(22)}$. As vantagens da utilização desse sistema foram amplamente discutidas ${ }^{(23)}$, e ele foi utilizado, também, no estudo de tradução e validação da ODM, para aplicação em nosso meio ${ }^{(17)}$. Em seguida, foi feita uma média, obtendo-se, assim, os valores médios em Sten, para os dois fatores citados.

Dessa forma foi possível a análise dos resultados por meio dos escores médios obtidos pelos sujeitos deste estudo, para cada um dos dois fatores da ODM, utilizandose o Sistema Sten, permitindo uma discussão e considerações sobre o autoritarismo e a benevolência desses estudantes frente à doença mental e ao seu portador.

\section{RESULTADOS}

Os resultados para o autoritarismo e a benevolência, frente à doença e ao doente mental, de alunos iniciantes no Curso de Enfermagem, apontaram, respectivamente, 6,66 e 5,86, em valores Sten (Figura 1).

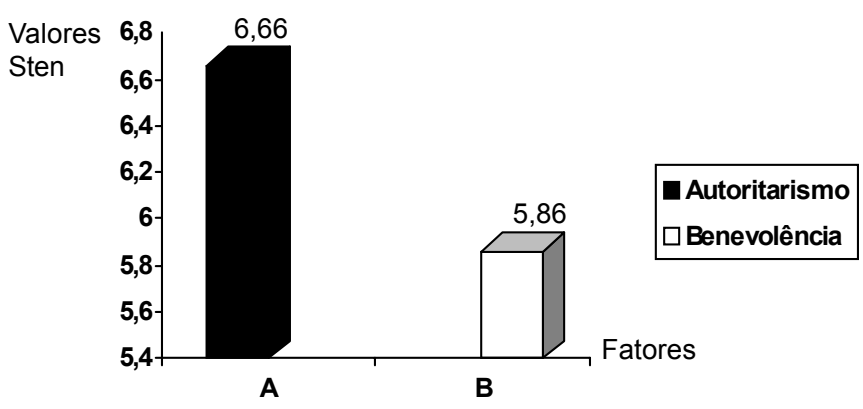

Figura 1 - Autoritarismo e benevolência frente à doença mental de alunos iniciantes no curso de enfermagem

\section{DISCUSSÃo}

O autoritarismo e a benevolência, de alunos iniciantes no curso de graduação em enfermagem, frente à doença mental e ao seu portador, foram estudados através da extração desses dois fatores pertencentes à ODM, por ter, em seus respectivos itens, amplitude suficiente à presunção deste estudo e também pelo fato de esses itens terem sido estudados isoladamente e agrupados segundo a adequação do seu conteúdo ao aspecto conceitual do fator ao qual iriam pertencer, quando a escala referida passou pelo processo de tradução e validação para o nosso meio ${ }^{(17)}$. Não se trata, portanto, de uma adaptação do instrumento, mas, sim, da utilização de parte dele com a manutenção de sua estrutura original, procedimento semelhante ao utilizado em um outro estudo ${ }^{(24)}$, onde, a partir da extração de alguns itens de duas escalas distintas, elaborou uma terceira, adequada a situação que pretendia pesquisar.

Os alunos participantes do estudo pertencem ao quadro discente de uma escola de enfermagem considerada modelo de ensino dessa profissão, localizada em uma região bem desenvolvida de um país com grande diversidade socioeconômica e que sofre seqüelas deixadas por uma política administrativa autoritária e restritiva, predominante durante muitos anos, influenciando desfavoravelmente a cultura e que, apenas recentemente, deixou de ser tão prevalente.

Dessa forma, esses alunos, filhos de pessoas influenciadas diretamente por essa política, não deixam de ser frutos de uma época que não facilitou em nada o desenvolvimento de técnicas, abordagens e formas de tratamento (humanos, inclusive) que objetivassem a recuperação e a inserção social da pessoa que sofre de transtornos psíquicos.

Esses aspectos, somados ao modo de entendimento do comportamento dessa pessoa (em sofrimento psíquico), pela população de forma geral, que não relaciona o modo agressivo ou estereotipado de se apresentar com os sintomas dos transtornos mentais, são fatores que podem justificar a atitude negativa, com conteúdo autoritário, apresentado pelos alunos, justificado pelo fato de não terem recebido ainda nenhuma instrução acadêmica nesse sentido e preservarem uma postura leiga semelhante à da maioria das pessoas ${ }^{(19)}$.

O protecionismo bondoso e paternalista, caracterizado por atitudes benevolentes frente ao portador de doença mental, é apresentado de forma adequada pelos alunos estudados, estando de acordo com resultados de outro estudo ${ }^{(20)}$, onde foi demonstrado que esses aspectos mantêm uma relação significativa com o grau de instrução, ou seja, as pessoas com um nível 
educacional melhor tendem a posturas menos moralistas e paternalistas.

No entanto, outros achados ${ }^{(16)}$ apontam os enfermeiros como os mais benevolentes, restritivos e discriminadores entre vários profissionais de saúde estudados, daí a necessidade de uma atenção especial à tendência atitudinal que apresentam os alunos sujeitos deste estudo, fatores esses que podem ser bem trabalhados nas disciplinas das áreas de Enfermagem Psiquiátrica e de Enfermagem em Saúde Mental.

Esses aspectos têm importância ímpar devido ao fato de que o aluno de enfermagem, recebendo uma instrução acadêmica, tenha oportunidades de oferecer assistência de forma holística ao ser humano e que seu treinamento, também, enfoque o meio ambiente de trabalho, cujas influências negativas tenham chances de ser trabalhadas. Quando esse aluno tornar-se um profissional, certamente poderá adequar-se melhor a um trabalho multiprofissional e, seguramente, terá melhores condições para discernir o papel que tem para organizar o ambiente, que é peculiar da enfermagem, e o autoritarismo proveniente do exercício desse papel, tendo, assim, tranqüilidade para agir de forma terapêutica. Atitudes firmes e benevolentes, no sentido negativo, influenciam desfavoravelmente tanto na abordagem da pessoa em sofrimento psíquico, quanto nas próprias funções ocupacionais do enfermeiro psiquiátrico, o que implica ser ele o profissional da equipe multidisciplinar com a tarefa de manutenção da ordem e do bom funcionamento do serviço ${ }^{(17,25)}$. Nesse sentido, as atitudes firmes e terapêuticas que oferecem ao paciente noções

\section{REFERÊNCIAS BIBLIOGRÁFICAS}

1. Rocha RM. Enfermagem psiquiátrica: que papel é este? Rio de Janeiro: Instituto Franco Basaglia/Editora Te Corá;1994.

2. Fernandes JD. O ensino de enfermagem psiquiátrica no Brasil. [dissertação]. Salvador(Ba): Universidade Federal da Bahia; 1982.

3. Matheney R, Topalis M. Enfermeria psiquiátrica. México: Interamericana; 1962.

4. Minzoni MA. Assistência ao doente mental. Ribeirão Preto (SP): Gráfica Guarani; 1974.

5. Irving S. Enfermagem psiquiátrica básica. Rio de Janeiro (RJ): Interamericana; 1979

6. Stefanelli M. Relacionamento terapêutico enfermeirapaciente. Rev Esc Enfermagem USP 1983; 17(1):39-45.

7. Kyes J, Hofling C. Conceitos básicos de enfermagem psiquiátrica. Rio de Janeiro (RJ): Interamericana; 1985.

8. Taylor CM. Fundamentos de enfermagem psiquiátrica de Mereness. Porto Alegre: Artes Médicas; 1992.

9. Travelbee J. Intervention en enfermeria psiquiátrica. Cali: OMS; 1979. de limite e oportunidades de confronto do seu comportamento impróprio com a realidade, se não forem aplicadas adequadamente, tornam-se autoritárias e repressoras, e as atitudes benevolentes tornam-se paternalistas, não contribuindo em nada para a recuperação dessa pessoa.

Pode-se concluir que uma relação professor/ aluno, principalmente durante o desenvolvimento das atividades práticas das disciplinas, permite que esse professor sirva de modelo profissional para o aluno, situação essa plenamente possível na escola de enfermagem onde o estudo foi realizado. Dessa forma, os alunos têm mais oportunidades de trabalhar suas atitudes autoritárias e paternalistas, observando o modo como o professor se relaciona com o paciente. Esses aspectos devem se incluir nos projetos das disciplinas citadas.

O modo como uma disciplina é estruturada e como seus docentes desenvolvem sua estratégia didática é também um fator relevante, e atitudes como as apresentadas devem ser consideradas no momento de seu planejamento, pois, só assim, serão efetivamente trabalhadas.

O conteúdo das disciplinas e as estratégias didáticas para atingir os seus objetivos são fatores relevantes, e podem ser desenvolvidos a partir de dados obtidos dos alunos, relativos à situação que irão vivenciar como aprendizes. Estudos prévios como este são particularmente importantes no sentido de oferecer subsídios para isso e aumentar a chance de formação de um profissional mais completo.

10. Rodrigues RM. Enfermagem compreendida como vocação e sua relação com as atitudes dos enfermeiros frente às condições de trabalho. Rev Latino-am Enfermagem 2001 novembro-dezembro; 9(6):76-82.

11. Miranda CML. Internação psiquiátrica e reabilitação psicossocial. J Bras Psiquiatr 1998; 47(9):437-9.

12. Rodrigues CRC. Comparacion de actitudes de estudiantes de medicina braileños y españoles hacia la enfermedad mental. Actas Luso - Españoles de Neurologia, Psiquiatria y Ciências Afines 1992; 20(1):30-41.

13. Daniel LF. Atitudes interpessoais em enfermagem. São Paulo (SP): EPU; 1983.

14. Simões N. Da metalinguagem profissinal à elaboração de um vocabulário técnico científico na área de enfermagem. [tese]. Ribeirão Preto (SP): Escola de Enfermagem de Ribeirão Preto/USP; 1989.

15. Fishbein M, Ajzen I, Belif, attitude, intention and behavior: an introdution to theory and research. Massachusets: Addison - Wesley; 1975.

16. Nunnaly JC Jr. Popular concepts of mental health, theyr development and change. New York: Holt, Rinehart \& Winston; 1961. 
17. Rodrigues CRC. Atitudes frente a doença mental: estudo transversal de uma amostra deprofissionais da saúde. [tese]. Ribeirão Preto(SP): Faculdade de Medicina de Ribeirão Preto/ USP; 1983.

18. Hood RW Jr. Dogmatism and opinions about mental illness. Psychol Rep 1973; 32:1283-90.

19. Alessi NP, Silva GB, Ferreira Santos CA. O doente mental visto pela população de um município paulista. Neurobiologia 1978; 31(3):387-400.

20. Cohen J, Struening EL. Opinions about mental illness in the personnel of two large mental hospitals. J Abnormal Soc Psychol 1962; 64(5):349-60.

21. Struening EL, Cohen J. Fatorial invariance and other psychometric characteristics of five opinions about mental illness fators. Educ Psychol Measure 1963; 23(3):289-98.

22. Canfield AA. The sten scale: a modified C - scale. Educ Psychol Measure 1951; 11:295-7.

23. Lyman HB, Derived scores. In: Lyman HB. Test scores and what they mean. New Jersey: Prentice - Hall; 1963. p.90-136. 24. Ishara S. Psicoterapia de grupo em hospital dia: proposição de uma metodologia de estudo. [dissertação]. Ribeirão Preto (SP): Faculdade de Medicina de Ribeirão Preto/USP; 1996.

25. Meyer LM. Comparison of atitudes toward mental patients of junior and senior nursing student and theyr universit peers. Nurs Res 1973; 17(30):242-5. 